\title{
Data-enabled predictive control for grid-connected power converters
}

\section{Conference Paper}

Author(s):

Huang, Linbin; Coulson, Jeremy; Lygeros, John (i); Dörfler, Florian (D)

Publication date:

2019-12

Permanent link:

https://doi.org/10.3929/ethz-b-000390823

\section{Rights / license:}

In Copyright - Non-Commercial Use Permitted

Originally published in:

https://doi.org/10.1109/CDC40024.2019.9029522 


\title{
Data-Enabled Predictive Control for Grid-Connected Power Converters
}

\author{
Linbin Huang, Jeremy Coulson, John Lygeros and Florian Dörfler
}

\begin{abstract}
We apply a novel data-enabled predictive control (DeePC) algorithm in grid-connected power converters to perform safe and optimal control. Rather than a model, the DeePC algorithm solely needs input/output data measured from the unknown system to predict future trajectories. We show that the DeePC can eliminate undesired oscillations in a grid-connected power converter and stabilize an unstable system. However, the DeePC algorithm may suffer from poor scalability when applied in high-order systems. To this end, we present a finite-horizon output-based model predictive control (MPC) for grid-connected power converters, which uses an $\mathrm{N}$-step auto-regressive-moving-average (ARMA) model for system representation. The ARMA model is identified via an $N$-step prediction error method (PEM) in a recursive way. We investigate the connection between the DeePC and the concatenated PEM-MPC method, and then analytically and numerically compare their closed-loop performance. Moreover, the PEM-MPC is applied in a voltage source converter based HVDC station which is connected to a two-area power system so as to eliminate low-frequency oscillations. All of our results are illustrated with high-fidelity, nonlinear, and noisy simulations.
\end{abstract}

\section{INTRODUCTION}

The penetration of power-electronic devices in modern power systems is ever-increasing due to the development of renewable energy, microgrids, high-voltage direct-current (HVDC) transmission systems, etc. [1], [2]. Conventionally, the control structure of power converters is designed according to engineering experience and the corresponding control gain tuning is based on iterative trial-and-error methods. Also, lots of effort has been put into the modeling of power converters, which provides insights into the system dynamics and criteria for control gain tuning [3]-[5].

However, these approaches heavily rely on rich engineering experience and lack systematicness. In addition, the control structure generally assumes a stiff power grid and may present poor robustness against variable grid conditions. For example, the most widely-used control structure, which consists of a phase-locked loop (PLL) and a current control loop, can become unstable when the power converter is connected to a weak grid with high grid impedance (or equivalently, low short-circuit ratio) [6]-[8].

Even though offline design and analysis (based on a nominal model) can be conducted to determine an optimal control parameter set, optimal performance can rarely be achieved

L. Huang is with the College of Electrical Engineering at Zhejiang University, Hangzhou, China, and the Department of Information Technology and Electrical Engineering at ETH Zürich, Switzerland. (Email: huanglb@zju.edu.cn)

J. Coulson, J. Lygeros and F. Dörfler are with the Department of Information Technology and Electrical Engineering at ETH Zürich, Switzerland. (Emails: jcoulson@control.ee.ethz.ch, jlygeros@ethz.ch, dorfler@ethz.ch)

This research was supported by ETH Zürich Funds. during online operation because (i) the real parameters of the power converter are hard to obtain due to different operation conditions and manufacturing inaccuracy; (ii) sometimes the underlying algorithms for the converter are designed by another manufacturer and are not obtainable, i.e., some part of the converter system is unknown; (iii) the power grid is generally an unknown system from power converter side which significantly affects the dynamic performance; and (iv) the offline design generally employs a constant power grid model (which in most cases is assumed to be an infinite bus) for the power converter, yet the real power grid is variable.

Normally, these problems are handled using robust or adaptive methods [8], [9]. However, these methods are still model-based, result in complex controllers, and suffer from scalability problems for large and uncertain (or even partially unknown) models. Inspired by recent advances in machine learning and artificial intelligence, recent control approaches entirely circumvent such model-based solutions in favor of data-driven approaches [10]-[12].

In this paper, we use a novel Data-enabled Predictive Control (DeePC) algorithm to compute optimal and safe control policies for grid-connected power converters, which uses real-time feedback to drive the unknown system along a desired (i.e., optimal and constrained) trajectory [13]. The DeePC algorithm presented in [13] relies on behavioural system approach [14]-[17]. Instead of using a parametric model for system representation, the approach in [14]-[17] describes the input/output behaviour of the system through the subspace of the signal space in which trajectories live.

However, when applied in large-scale systems, e.g., in the case of power transmision oscillation damping [18], [19], the optimal regulation problem in DeePC may suffer from poor scalability due to its high dimension. To this end, we use a finite-horizon output-based model predictive control (MPC) for grid-connected power converters, wherein the unknown system is represented by an $N$-step auto-regressive-movingaverage (ARMA) model and identified via least-square $N$ step prediction error method (PEM). The PEM can be solved in a recursive way which enables an iterative calculation and possible online implementation. We will show that this PEMMPC method is scalable for large-scale unknown systems, and analytically discuss how it is related to DeePC.

The remainder of this paper is organized as follows: in Section II we provide an overview for the DeePC approach. Section III discusses how the concatenated PEM-MPC is related to DeePC and then presents their applications in a grid-connected power converter. In Section IV we apply the PEM-MPC in a two-area power system which contains one HVDC station. We conclude the paper in Section V. 


\section{Data-Enabled Predictive Control}

\section{A. Preliminaries and Notation}

For an unknown discrete-time LTI system that has $m$ inputs and $p$ outputs, we denote by $u_{t} \in \mathbb{R}^{m}$ the input vector of the system at time $t \in \mathbb{Z}_{\geq 0}$ and $y_{t} \in \mathbb{R}^{p}$ the output vector at time $t \in \mathbb{Z}_{\geq 0}$, where $\mathbb{Z}_{\geq 0}$ is the discrete-time axis. Let $u=$ $\operatorname{col}\left(u_{1}, u_{2}, \ldots\right)$ and $y=\operatorname{col}\left(y_{1}, y_{2}, \ldots\right)$ be respectively the input and output trajectories whose dimensions can be inferred from the context, where $\operatorname{col}\left(a_{1}, \ldots, a_{i}\right):=\left[a_{1}^{\top} \cdots a_{i}^{\top}\right]^{\top}$.

Let $L, T \in \mathbb{Z}_{\geq 0}$ and $T \geq L$. The trajectory $u \in \mathbb{R}^{m T}$ is persistently exciting of order $L$ if the Hankel matrix

$$
\mathscr{H}_{L}(u):=\left[\begin{array}{cccc}
u_{1} & u_{2} & \cdots & u_{T-L+1} \\
u_{2} & u_{3} & \cdots & u_{T-L+2} \\
\vdots & \vdots & \ddots & \vdots \\
u_{L} & u_{L+1} & \cdots & u_{T}
\end{array}\right]
$$

is of full row rank, i.e., the signal $u$ is sufficiently long and sufficiently rich.

Consider the following $n$-order discrete-time LTI system (minimal representation):

$$
\left\{\begin{array}{l}
x_{t+1}=A x_{t}+B u_{t} \\
y_{t}=C x_{t}+D u_{t}
\end{array}\right.
$$

where $A \in \mathbb{R}^{n \times n}, B \in \mathbb{R}^{n \times m}, C \in \mathbb{R}^{p \times n}, D \in \mathbb{R}^{p \times m}$, and $x_{t}$ is the state of the system at time $t \in \mathbb{Z}_{\geq 0}$.

The lag of the system in (2) is defined by the smallest integer $\ell \in \mathbb{Z}_{\geq 0}$ so that the observability matrix

$$
\mathscr{O}_{\ell}(A, C):=\operatorname{col}\left(C, C A, \ldots, C A^{\ell-1}\right)
$$

has rank $n$.

Let $T_{\text {ini }}, N \in \mathbb{Z}_{\geq 0}$ such that $T \geq(m+1)\left(T_{\text {ini }}+N+n\right)-1$. Consider an input trajectory $u^{\mathrm{d}}$ and an output trajectory $y^{\mathrm{d}}$ (both are of length $T \in \mathbb{Z}_{\geq 0}$, i.e., $u^{\mathrm{d}} \in \mathbb{R}^{m T}$ and $y^{\mathrm{d}} \in \mathbb{R}^{p T}$ ) measured from the $n$-order unknown system (2) such that $u^{\mathrm{d}}$ is persistently exciting of order $T_{\mathrm{ini}}+N+n$. Here we use the superscript $d$ to indicate that these two trajectories are data sets measured from the unknown system. We use $u^{\mathrm{d}}$ and $y^{\mathrm{d}}$ to construct the Hankel matrices $\mathscr{H}_{T_{\mathrm{ini}}+N}\left(u^{\mathrm{d}}\right)$ and $\mathscr{H}_{T_{\text {ini }}+N}\left(y^{\mathrm{d}}\right)$, which are further partitioned into two parts as

$$
\left[\begin{array}{c}
U_{P} \\
U_{f}
\end{array}\right]:=\mathscr{H}_{T_{\mathrm{ini}}+N}\left(u^{\mathrm{d}}\right), \quad\left[\begin{array}{c}
Y_{P} \\
Y_{f}
\end{array}\right]:=\mathscr{H}_{T_{\mathrm{ini}}+N}\left(y^{\mathrm{d}}\right),
$$

where $\quad U_{P} \in \mathbb{R}^{m T_{\mathrm{ini}} \times\left(T-T_{\mathrm{ini}}-N+1\right)}, \quad U_{f}$ $\mathbb{R}^{m N \times\left(T-T_{\mathrm{ini}}-N+1\right)}, \quad Y_{P} \in \mathbb{R}^{p T_{\mathrm{ini}} \times\left(T-T_{\mathrm{ini}}-N+1\right)}$ and $Y_{f} \in \mathbb{R}^{p N \times\left(T-T_{\mathrm{ini}}-N+1\right)}$.

According to the behavioral system theory [15], $\operatorname{col}\left(u_{\text {ini }}, y_{\text {ini }}, u, y\right)$ is a trajectory of (2) if and only if there exist $g \in \mathbb{R}^{T-T_{\mathrm{ini}}-N+1}$ such that

$$
\left[\begin{array}{c}
U_{P} \\
Y_{P} \\
U_{f} \\
Y_{f}
\end{array}\right] g=\left[\begin{array}{c}
u_{\mathrm{ini}} \\
y_{\mathrm{ini}} \\
u \\
y
\end{array}\right] .
$$

The trajectory $\operatorname{col}\left(u_{\text {ini }}, y_{\text {ini }}\right)$ can be thought of as an initial condition for the trajectory $\operatorname{col}\left(u_{\mathrm{ini}}, y_{\mathrm{ini}}, u, y\right)$ and $\operatorname{col}(u, y)$ as a future trajectory departing from this initial condition. If $T_{\text {ini }} \geq \ell$, the future output trajectory $y$ is uniquely determined through (4) for every given input trajectory $u$.

\section{B. Review of DeePC}

Instead of learning a parametric system representation through system identification, the DeePC attempts to learn the system's behaviour and computes optimal control inputs using past data measured from the unknown system. Moreover, input/output constraints can be conveniently incorporated to ensure safety, described as follows.

After using the input/output trajectory $\operatorname{col}\left(u^{\mathrm{d}}, y^{\mathrm{d}}\right)\left(u^{\mathrm{d}} \in\right.$ $\mathbb{R}^{m T}$ and $y^{\mathrm{d}} \in \mathbb{R}^{p T}$ ) to construct the Hankel matrices in (3), DeePC solves the following optimization problem at every sampling time to get the optimal future control inputs

$$
\begin{aligned}
& \min _{g, u \in \mathcal{U}, y \in \mathcal{Y}}\|u\|_{R}^{2}+\|y-r\|_{Q}^{2}+\lambda_{g}\|g\|_{2}^{2} \\
& \text { s.t. }\left[\begin{array}{c}
U_{P} \\
Y_{P} \\
U_{f} \\
Y_{f}
\end{array}\right] g=\left[\begin{array}{c}
u_{\text {ini }} \\
y_{\text {ini }} \\
u \\
y
\end{array}\right],
\end{aligned}
$$

(DeePC)

where $\mathcal{U} \subseteq \mathbb{R}^{m N}$ and $\mathcal{Y} \subseteq \mathbb{R}^{p N}$ are the input and output constraint sets, $R \in \mathbb{R}^{m N \times m N}$ is the control cost matrix (positive definite), $Q \in \mathbb{R}^{p N \times p N}$ is the output cost matrix (positive semidefinite), $\lambda_{g} \in \mathbb{R}_{\geq 0}$ is the regularization parameter, $r \in \mathbb{R}^{p N}$ is the reference vector for the output signals, $N$ is the prediction horizon, and $\operatorname{col}\left(u_{\text {ini }}, y_{\text {ini }}\right)$ consists of the most recent input/output trajectory of (2). The norm $\|a\|_{X}^{2}$ of the vector $a$ denotes the quadratic form $a^{\top} X a$.

We note that a two-norm penalty on $g$ is included in the cost function as a regularization term to avoid overfitting. More precisely, when the data samples of the Hankel matrix and the online measurements $y_{\text {ini }}$ are corrupted by stochastic disturbances, a two-norm regularization on $g$ coincides with an analogous two-norm distributional robustness of the DeePC optimization problem [20]. The DeePC involves solving the optimization problem (DeePC) in a receding horizon manner [13], that is, after calculating the optimal control input sequence $u^{\star}$, we apply $\left(u_{t}, \ldots, u_{t+s}\right)=\left(u_{0}^{\star}, \ldots, u_{s}^{\star}\right)$ to the system for some $s \leq N-1$ time steps, update $\operatorname{col}\left(u_{\text {ini }}, y_{\text {ini }}\right)$ to the most recent input/output measurements and then set $t$ to $t+s+1$ for the DeePC algorithm.

\section{From DEEPC TO PEM-MPC}

\section{A. Scalability of DeePC}

The DeePC approach provides a safe and optimal solution to the regulation problem by solely using the measured data from the unknown system. However, when applied in highorder systems, the DeePC may suffer from poor scalability because the dimension of the decision variable $g$ (which is $T-T_{\mathrm{ini}}-N+1$ ) depends on the length of data $T$ to construct the Hankel matrix. In other words, the optimization problem in (DeePC) is of high dimension when choosing a long sequence of data to possibly eliminate the impacts of measurement noise. 
To remove $g$ from the constraint and ensure the scalability of the optimization problem in (DeePC), we consider a subsequent system identification and model predictive control whose decision variables are $u$ and $y$.

\section{B. Model Predictive Control}

In what follows, we consider the following finite-horizon output-based MPC problem

$$
\begin{aligned}
& \min _{u \in \mathcal{U}, y \in \mathcal{Y}}\|u\|_{R}^{2}+\|y-r\|_{Q}^{2} \\
& \text { s.t. } y=K\left[\begin{array}{c}
u_{\text {ini }} \\
y_{\text {ini }} \\
u
\end{array}\right],
\end{aligned}
$$

where the decision variables $u$ and $y$ are the control inputs and measurement outputs over the prediction horizon, and $K \in \mathbb{R}^{p N \times\left(m T_{\mathrm{ini}}+p T_{\mathrm{ini}}+m N\right)}$ is the $N$-step transition matrix predicting how future outputs of the system are determined by the initial input/output data and the future inputs. Note that the optimization problem (MPC) is solved in a receding horizon manner resulting in an online feedback control.

Since the decision variable $g$ in (DeePC) does not appear in (MPC), solving (MPC) has much less computational burden than solving (DeePC), especially when the Hankel matrix has high column number. On the other hand, (MPC) depends on an explicit predictive model given by the transition matrix $K$ in the equality constraint.

\section{Prediction Error System Identification}

Observe that the predictive model in (MPC) is an $N$-step ARMA model for the discrete-time LTI system mapping past inputs and outputs $\operatorname{col}\left(u_{\text {ini }}, y_{\text {ini }}\right)$ as well as future inputs $u$ to future outputs $y$. In particular, for the output vector at discrete time $i(i \in\{1, \ldots, N\})$, i.e., $y_{i}$, we have

$$
y_{i}=K_{i} \varphi
$$

where $K_{i} \in \mathbb{R}^{p \times\left(m T_{\mathrm{ini}}+p T_{\mathrm{ini}}+m N\right)}$ is the $i$ th block row of $K$ and $\varphi=\operatorname{col}\left(u_{\text {ini }}, y_{\text {ini }}, u\right)$.

Given past measurements of $y_{i}$ and $\varphi$, the transition matrix $K_{i}$ can be computed offline through system identification. In the absence of measurement noise, $K_{i}$ can be computed exactly with $m T_{\text {ini }}+p T_{\text {ini }}+m N$ linearly independent measurements of $\varphi$ and associated $y_{i}$. However, measurement noise will significantly affect the accuracy of this approach. A standard solution to remedy this problem and to eliminate the effects of the noise is to use a larger data set and apply a least-square $N$-step PEM minimizing

$$
\min _{K_{i}} \sum_{j=1}^{N_{\text {trj }}}\left\|y_{i(j)}-K_{i} \varphi_{(j)}\right\|_{2}^{2},
$$

(PEM)

where $N_{\text {trj }}>m T_{\text {ini }}+p T_{\text {ini }}+m N$ is the number of measured trajectories, and $y_{i(j)}$ and $\varphi_{(j)}$ belong to the $j$ th trajectory. Indeed, the subsequent combination of PEM and MPC is a standard approach to model-based control that has proved itself in many applications throughout academia and industry [21], [22].
As an alternative to the batch optimization approach (PEM) combining all the measured trajectories to solve for $K$ in one step, $K$ can be obtained by adopting the recursive least-square algorithm [23]. The recursive algorithm is not only more scalable for large data sets, but it could possibly also be applied online to adapt the model $K$ used in (MPC) to a variable environment. Moreover, the size of $K$ is solely related to $T_{\text {ini }}$ and $N$ and independent of the size of the data set, which leads to a smaller optimization problem size than (DeePC) when applied to high-order systems.

\section{Relation of DeePC, MPC, and PEM}

In the following, we will relate the subsequent system identification through (PEM) and model-based control through (MPC) to the data-driven (DeePC) strategy. In a first step, observe that the least-square solution for (PEM) can be expressed in closed form as

$$
K_{i}=\left[\begin{array}{lll}
y_{i(1)} & \cdots & y_{i\left(N_{\mathrm{trj}}\right)}
\end{array}\right]\left[\begin{array}{lll}
\varphi_{(1)} & \cdots & \varphi_{\left(N_{\mathrm{trj}}\right)}
\end{array}\right]^{+},
$$

where the Moore-Penrose pseudoinverse of a matrix $X$ is denoted by $X^{+}$.

The transition matrix $K$ can then be obtained as

$$
K=\left[\begin{array}{lll}
y_{(1)} & \cdots & y_{\left(N_{\mathrm{trj}}\right)}
\end{array}\right]\left[\begin{array}{lll}
\varphi_{(1)} & \cdots & \varphi_{\left(N_{\mathrm{trj}}\right)}
\end{array}\right]^{+} .
$$

Since every column in the constructed Hankel matrix (3) is one trajectory measured from the unknown system, $K$ can also be calculated by using the Hankel matrix (3) by

$$
K=Y_{f}\left[\begin{array}{l}
U_{P} \\
Y_{P} \\
U_{f}
\end{array}\right]^{+} .
$$

By combining (8) with $y=K \varphi$ and $y=Y_{f} g$ one obtains

$$
g=\left[\begin{array}{c}
U_{P} \\
Y_{P} \\
U_{f}
\end{array}\right]^{+}\left[\begin{array}{c}
u_{\text {ini }} \\
y_{\text {ini }} \\
u
\end{array}\right],
$$

which equals the solution of the optimization problem

$$
\begin{array}{ll}
\min _{g} & \|g\|_{2}^{2} \\
\text { s.t. } & {\left[\begin{array}{c}
U_{P} \\
Y_{P} \\
U_{f}
\end{array}\right] g=\left[\begin{array}{c}
u_{\text {ini }} \\
y_{\text {ini }} \\
u
\end{array}\right] .}
\end{array}
$$

Observe that the optimization problem (LN) seeks the leastnorm solution to the equality constraint. Thus, we refer to it as the least-norm $(\mathbf{L N})$ problem. For Hankel matrix data, the solution $g^{\prime \star}$ of (LN) is related to the solution of the leastsquares (PEM) problem $K^{\star}$ by $y=Y_{f} g^{\prime \star}=K^{\star} \varphi$. It can also be derived using subspace identification methods [17]. We summarize this observation below.

Lemma 3.1 (PEM and LN): Consider the least-norm problem $(\mathrm{LN})$ with Hankel matrix $\operatorname{col}\left(U_{P}, Y_{P}, U_{f}\right)$. Consider the least-square $N$-step prediction error method optimization problem (PEM), and assume that its data $y_{i(j)}$ and $\varphi_{(j)}$ is arranged into the Hankel matrix $\operatorname{col}\left(U_{P}, Y_{P}, U_{f}, Y_{f}\right)$. Then the solution $K^{\star}$ of (PEM) and the solution $g^{\prime \star}$ of (LN) are related by the equation $K^{\star} \varphi=Y_{f} g^{\prime \star}$. 
Observe that if the (LN) solution $g^{\prime \star}$ is used as predictive model (equality constraint) of the (MPC) problem by setting $y=Y_{f} g^{\prime \star}$ (where $g^{\prime \star}$ is a function of $u$ ), then the subsequent concatenation of the (MPC) and the (LN) (or equivalently (PEM) by Lemma 3.1) optimization problems reads as follows:

$$
\begin{aligned}
& \min _{u^{\prime} \in \mathcal{U}, y^{\prime} \in \mathcal{Y}}\left\|u^{\prime}\right\|_{R}^{2}+\left\|y^{\prime}-r\right\|_{Q}^{2} \\
& \text { s.t. } u^{\prime}=U_{f} g^{\prime \star}, y^{\prime}=Y_{f} g^{\prime \star}, \\
& \text { where } g^{\prime \star}=\underset{g^{\prime}}{\arg \min }\left\|g^{\prime}\right\|_{2}^{2} \\
& \text { s.t. }\left[\begin{array}{c}
U_{P} \\
Y_{P} \\
U_{f}
\end{array}\right] g^{\prime}=\left[\begin{array}{c}
u_{\text {ini }} \\
y_{\text {ini }} \\
u^{\prime}
\end{array}\right] .
\end{aligned}
$$

(PEM-MPC)

Note that $g^{\prime \star}$ depends on the decision variable $u^{\prime}$ in (PEM-MPC). The inner problem of (PEM-MPC) is the system identification step through (LN) (or equivalently (PEM) by Lemma 3.1). The outer problem on the other hand is identical to the (MPC) problem. Let

$$
C(u, y, g)=\|u\|_{R}^{2}+\|y-r\|_{Q}^{2}+\lambda_{g}\|g\|_{2}^{2}
$$

be the combined cost taking into account both system performance (i.e., $\|u\|_{R}^{2}+\|y-r\|_{Q}^{2}$ ) and the complexity of $g$ (i.e., $\left.\lambda_{g}\|g\|_{2}^{2}\right)$. This is the true cost we wish to minimize when noise is present in the system as large entries of $g$ result in overfitting of the noisy trajectories in the Hankel matrix in (4) [13]. Since this cost appears in (DeePC), we can compare directly the performance of (PEM-MPC) and (DeePC) with respect to this cost. We present the comparison below.

Lemma 3.2 (PEM-MPC and DeePC): Consider the optimal solution $\left(u^{\prime \star}, y^{\prime \star}, g^{\prime \star}\right)$ of the concatenated (PEM-MPC) problem and the optimal solution $\left(u^{\star}, y^{\star}, g^{\star}\right)$ of the (DeePC) problem. It holds that

$$
C\left(u^{\star}, y^{\star}, g^{\star}\right) \leq C\left(u^{\prime \star}, y^{\prime \star}, g^{\prime \star}\right) .
$$

That is, (DeePC) achieves a cost less or equal to the cost of the concatenated (PEM-MPC) with respect to (10).

Proof: Observe that any feasible point $\left(u^{\prime}, y^{\prime}, g^{\prime \star}\right)$ of (PEM-MPC) is also a feasible point of (DeePC). Since $g^{\prime \star}$ is a particular $g$ that satisfies the constraints of (DeePC) then the feasible set of (PEM-MPC) is a subset of (DeePC). Hence, it holds that $C\left(u^{\star}, y^{\star}, g^{\star}\right) \leq C\left(u^{\prime \star}, y^{\prime \star}, g^{\prime \star}\right)$.

In other words, the DeePC presents better performance than the MPC formulated in (MPC) if $K$ is obtained by (PEM). On the other hand, the MPC problem in (MPC) has the advantage that it doesn't contain the decision variable $g$ and therefore has lower computational burden, which make it scalable to high-order systems. In the next section we numerically observe that the gap between (DeePC) and (PEM-MPC) presented in Lemma 3.2 can actually be made smaller by an appropriate choice of regularization $\lambda_{g}$.

\section{E. Applications to a Grid-Connected Power Converter}

In this section, we use the DeePC and the PEM-MPC to perform optimal control for a grid-connected power converter as shown in Fig.1. The power converter together with the power grid is a black-box system from the view of the

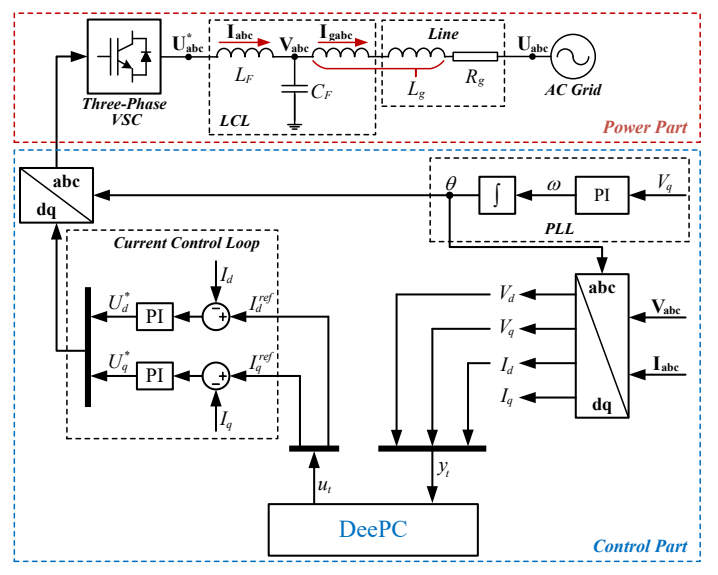

Fig. 1. One-line diagram of a grid-connected power converter.

DeePC/PEM-MPC. The control part of the converter consists of a PLL, a current control loop and coordinate transformation blocks [4], [24]. The sampling frequency of the DeePC and PEM-MPC is $1 \mathrm{kHz}$. The other system parameters can be found in the extended version of this paper [25].

When the converter is connected to a strong grid that features low grid-side impedance, it will present anticipated dynamic performance. However, if the converter is connected to a weak grid that has high grid-side impedance, e.g., $L_{g}=0.35$ p.u., the PLL will have significant interaction with the current control loop as well as the grid impedance, which may result in instabilities [6], [8].

We choose $I_{d}^{\text {ref }}$ and $I_{q}^{\text {ref }}$ to be the control inputs, and the measured outputs (with noise) from the black-box system are $V_{d}, V_{q}$ and $I_{d}$. Since the DeePC has no information about the black-box system, $T_{\mathrm{ini}}$ is chosen to be sufficiently large $\left(T_{\mathrm{ini}}=40\right)$ to meet $T_{\mathrm{ini}} \geq \ell$. Before $t=0 \mathrm{~s}$, white noise signals are injected into the system via $I_{d}^{\text {ref }}$ and $I_{q}^{\text {ref }}$ so as to obtain the Hankel matrix.

Fig.2 plots the time-domain responses of the power converter when the DeePC is applied. By choosing $T=500$, the DeePC effectively eliminates the voltage and current oscillations when activated at $t=0.2 \mathrm{~s}$, and presents robustness when $L_{g}$ is changed from $0.34 \mathrm{p} . \mathrm{u}$. to $0.35 \mathrm{p} . \mathrm{u}$. at $t=0.7 \mathrm{~s}$, and to 0.5 p.u. at 1.0s. Note the Hankel matrix is not updated during the disturbances.

When choosing $T=330$ in the DeePC, the oscillations are obviously eliminated as well after the DeePC is activated. However, the voltage and current signals are slightly oscillating after $L_{g}$ is changed from 0.35 p.u. to $0.5 \mathrm{p} . \mathrm{u}$. at $1.0 \mathrm{~s}$. This is because the trajectory $\operatorname{col}\left(u^{\mathrm{d}}, y^{\mathrm{d}}\right)$ in this case contains less information of the system than that with $T=500$, and thus the prediction is more strongly affected by the measurement noise when solving the DeePC. Choosing a larger $T$ is a convenient way to resolve this problem, yet it results in a higher dimension of $g$ and thus higher computational burden in solving (DeePC), which may not be scalable to high- $\ell$ systems as discussed before.

Fig.2 also displays the relationship between $T$ and the time-domain cost (from $t=0.2 \mathrm{~s}$ to $t=1.4 \mathrm{~s}$ ) to illustrate how different values of $T$ affect the performance, where the 

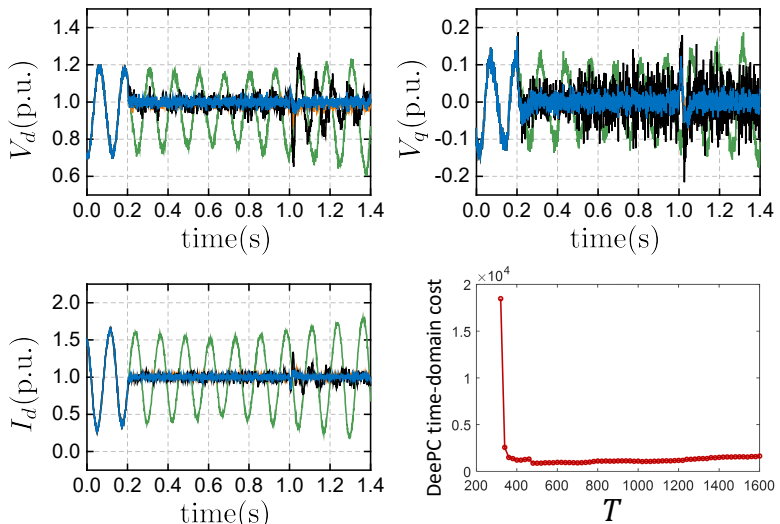

Fig. 2. Time-domain responses and time-domain cost. The DeePC/PEMMPC is activated at $t=0.2 \mathrm{~s}$. - PEM-MPC; - DeePC $(T=500)$; DeePC ( $T=330)$; — without DeePC or PEM-MPC; — time-domain cost with different $T$.
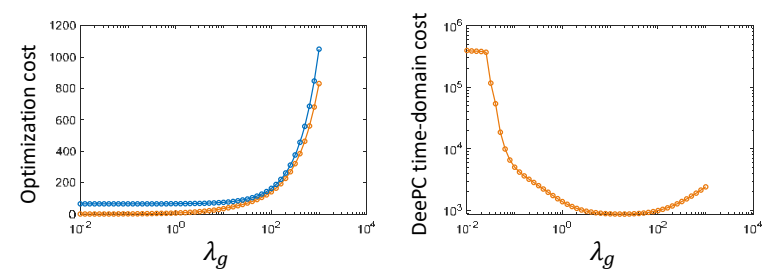

Fig. 3. Variations of the optimization cost and the time-domain cost with different values of $\lambda_{g}$. - DeePC; — PEM-MPC;

time-domain cost is $\left\|u_{\text {time }}\right\|_{R}^{2}+\left\|y_{\text {time }}-r\right\|_{Q}^{2}\left(u_{\text {time }}\right.$ and $y_{\text {time }}$ are the input and output trajectories measured from the closed-loop system, and $r$ is the reference for the outputs). It is shown that the time-domain cost sharply decreases with the increase of $T$ from 320 to 400 , and remain nearly the same (or slightly increases) if further increasing $T$.

For comparison, Fig. 2 plots the responses of the power converter when the PEM-MPC is used. Before $t=0 \mathrm{~s}$, the $N$-step transition matrix $K$ has been obtained during the converter's operation by recursively solving the PEM problem in (PEM) with 1500 trajectories.

At $t=0.2 \mathrm{~s}$, the PEM-MPC is activated, which effectively attenuates the voltage and current oscillations and stabilizes the system. Then, we test the robustness of the PEM-MPC by changing the grid-side inductance $L_{g}$ from $0.34 \mathrm{p} . \mathrm{u}$. to $0.35 \mathrm{p} . \mathrm{u}$. at $t=0.7 \mathrm{~s}$, and to $0.5 \mathrm{p} . \mathrm{u}$. at $1.0 \mathrm{~s}$. It can be seen that the system is stable under these two disturbances, and the current and voltage signals track the references with fast dynamics, that is, the PEM-MPC shows robustness in terms of parameter changes in the black-box system. Here $K$ is not updated during the two disturbances to test how an inaccurate model in $K$ affects the performance of the PEM-MPC.

The converter's responses without the DeePC or the PEMMPC are plotted by the green lines in Fig.2. The system is unstable with the voltage and current signals keeping oscillating, which endangers the power system operation.

Fig.3 displays how $\lambda_{g}$ affects the optimization cost of the system (setting $T=500$ ). The optimization costs of DeePC and PEM-MPC are respectively obtained by solving (DeePC) and (PEM-MPC) at $t=0.2 \mathrm{~s}$, and Fig.3 shows

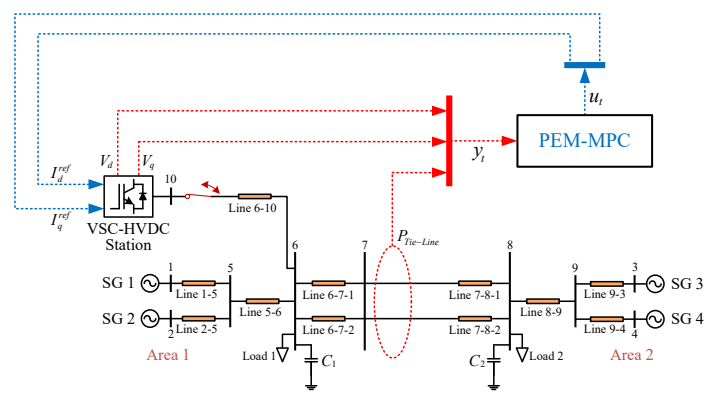

Fig. 4. One-line diagram of a three-phase two-area test system with integration of a VSC-HVDC station.

that DeePC outperforms PEM-MPC under any $\lambda_{g}$, which is consistent with Lemma 3.2. In addition, Fig.3 also plots how $\lambda_{g}$ affects the time-domain cost (from $t=0.2 \mathrm{~s}$ to $t=1.4 \mathrm{~s}$ ) of the DeePC closed-loop system, and shows that this cost dramatically decreases when increasing $\lambda_{g}$ from 0.01 to 20 before increasing again consistent with the theoretic predictions from distributional robustness [20].

\section{Application of PEM-MPC IN LARGE-SCALE SYSTEMS}

To illustrate the effectiveness of the PEM-MPC in largescale power systems, we now provide a detailed simulation study based on a nonlinear model of the three-phase two-area test system (the order of the system is $n=90$ ) in Fig.4. This test system consists of four synchronous generators (SGs) and one voltage source converter (VSC) HVDC station. The VSC-HVDC station is a large-capacity power converter with an $L C L$ filter, which in our case, employs the control scheme given in Fig.1. The system parameters can be found in the extended version of this paper [25].

This test system has one pair of weakly-damped inter-area modes and presents low-frequency oscillations, as caused by the fast exciters in the SGs as well as long transmission lines [26]. The system inputs/outputs for the PEM-MPC are depicted in Fig.4. Fig.5 plots the time-domain responses of the inter tie-line active power when different $T_{\text {ini }}$ and $N$ are adopted. The sampling time for PEM-MPC is $1 \mathrm{~ms}$. Note that the $N$-step transition matrix $K$ was calculated iteratively with 3000 trajectories before $t=0 \mathrm{~s}$.

Firstly, since the test system is of high order (with 90 state variables) and is a black-box system to the PEM-MPC, we choose a sufficiently large $T_{\mathrm{ini}}\left(T_{\mathrm{ini}}=200\right)$ to meet $T_{\mathrm{ini}} \geq \ell$, and the prediction horizon is chosen as $N=80$. It can be seen that the inter-area oscillation is well eliminated after the PEM-MPC is activated at $t=10 \mathrm{~s}$. Then, considering that the low-frequency oscillations are caused by the interactions among the SGs' rotors and thus can possibly be represented by an equivalent low-order system, $T_{\text {ini }}$ and $N$ are chosen smaller to test the performance of the PEM-MPC. Fig.5 shows that when $\left(T_{\text {ini }}, N\right)$ are chosen to be $(10,10)$ and even $(5,10)$, the PEM-MPC can still effectively attenuate the inter tie-line power oscillations. Also observe that with the decrease of $T_{\mathrm{ini}}$ and $N$, some slight oscillations still exist after the PEM-MPC is activated, which is caused by the 

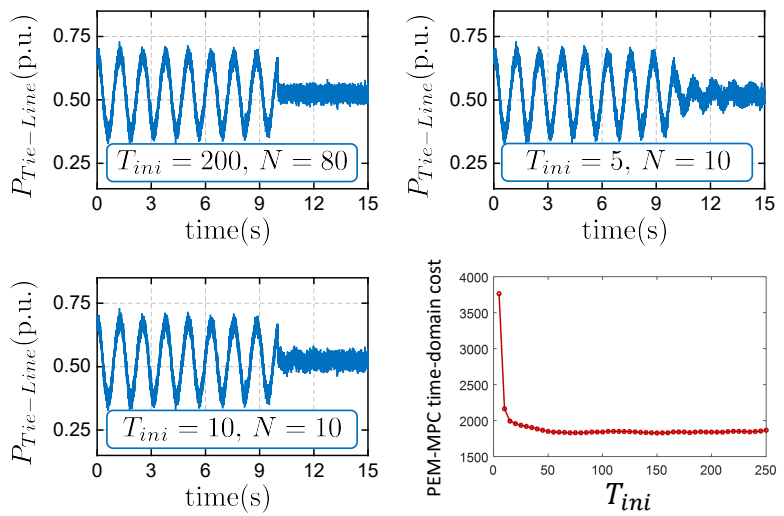

Fig. 5. Time-domain responses and time-domain cost. — Time-domain responses of the inter tie-line active power (with PEM-MPC activated at $t=10 \mathrm{~s}) ;$ - time-domain cost with different $T_{\mathrm{ini}}$.

model mismatch, i.e., a smaller size of $K$ may not be able to accurately represent the full-order model.

To further illustrate how the choice of $T_{\text {ini }}$ affects the overall performance, Fig.5 also plots the variations of the time-domain cost with different values of $T_{\mathrm{ini}}$ (from $t=$ $10 \mathrm{~s}$ to $t=14 \mathrm{~s})$. It shows that the time-domain cost is significantly reduced with the increase of $T_{\text {ini }}$ from 5 to 50 , but remains nearly the same if further increasing $T_{\text {ini }}$.

\section{CONCLusion}

We applied DeePC in grid-connected power converters to eliminate undesired oscillations caused by weak grid conditions. The DeePC has no information about the power converter or the power grid, and solely uses input/output data measured from the unknown system. We showed that the DeePC can stabilize an unstable system by performing optimal and constrained receding-horizon control. We illustrated that DeePC shows increased level of robustness and performance when considering a longer input/output data sequence to construct the Hankel matrix, which at the same time results in higher computational burden and thus possibly poor scalability especially in high-order systems. For this reason, we presented a concatenated PEM-MPC method as an alternative model-based, optimal, and constrained recedinghorizon control, wherein the PEM can be implemented in a recursive manner. We discussed the connection between DeePC and PEM-MPC, and formally showed that the DeePC outperforms PEM-MPC with regards to the cost in the optimization problem. We applied the PEM-MPC in a VSCHVDC station which is connected to a high-order power grid that contains four SGs, and showed that the PEM-MPC effectively eliminates the low-frequency oscillations caused by the interactions among multiple SGs.

\section{REFERENCES}

[1] F. Milano, F. Dörfler, G. Hug, D. Hill, and G. Verbic, "Foundations and challenges of low-inertia systems," in Power Systems Computation Conference (PSCC), Dublin, Ireland, 2018.

[2] D. E. Olivares, A. Mehrizi-Sani, A. H. Etemadi et al., "Trends in microgrid control," IEEE Trans. Smart Grid, vol. 5, no. 4, pp. 19051919, 2014.
[3] N. Pogaku, M. Prodanovic, and T. C. Green, "Modeling, analysis and testing of autonomous operation of an inverter-based microgrid," IEEE Trans. Power Electron., vol. 22, no. 2, pp. 613-625, 2007.

[4] L. Harnefors, "Modeling of three-phase dynamic systems using complex transfer functions and transfer matrices," IEEE Trans. Ind. Electron., vol. 54, no. 4, pp. 2239-2248, 2007.

[5] M. Cespedes and J. Sun, "Impedance modeling and analysis of gridconnected voltage-source converters," IEEE Trans. Power Electron., vol. 29, no. 3, pp. 1254-1261, 2014.

[6] B. Wen, D. Boroyevich, R. Burgos, P. Mattavelli, and Z. Shen, "Analysis of dq small-signal impedance of grid-tied inverters," IEEE Trans. Power Electron., vol. 31, no. 1, pp. 675-687, 2016.

[7] J. A. Suul, S. D'Arco, P. Rodríguez, and M. Molinas, "Impedancecompensated grid synchronisation for extending the stability range of weak grids with voltage source converters," IET Generation, Transmission \& Distribution, vol. 10, no. 6, pp. 1315-1326, 2016.

[8] L. Huang, H. Xin, Z. Wang et al., "An adaptive phase-locked loop to improve stability of voltage source converters in weak grids," in Power and Energy Society General Meeting (PESGM), Portland, OR, USA. IEEE, 2018, pp. 1-5.

[9] G. Weiss, Q.-C. Zhong, T. C. Green, and J. Liang, "H/sup/spl infin//repetitive control of dc-ac converters in microgrids," IEEE Transactions on Power Electronics, vol. 19, no. 1, pp. 219-230, 2004

[10] F. L. Lewis, D. Vrabie, and K. G. Vamvoudakis, "Reinforcement learning and feedback control: Using natural decision methods to design optimal adaptive controllers," IEEE Control Systems Magazine, vol. 32, no. 6, pp. 76-105, 2012.

[11] S. Dean, H. Mania, N. Matni, B. Recht, and S. Tu, "On the sample complexity of the linear quadratic regulator," arXiv preprint arXiv:1710.01688, 2017.

[12] R. Boczar, N. Matni, and B. Recht, "Finite-data performance guarantees for the output-feedback control of an unknown system," in 2018 IEEE Conference on Decision and Control (CDC). IEEE, 2018, pp. 2994-2999.

[13] J. Coulson, J. Lygeros, and F. Dörfler, "Data-enabled predictive control: In the shallows of the deepc," arXiv preprint arXiv:1811.05890, 2018.

[14] I. Markovsky, J. C. Willems, S. Van Huffel, and B. De Moor, Exact and approximate modeling of linear systems: A behavioral approach. SIAM, 2006, vol. 11.

[15] J. C. Willems, P. Rapisarda, I. Markovsky, and B. L. De Moor, "A note on persistency of excitation," Systems \& Control Letters, vol. 54, no. 4, pp. 325-329, 2005.

[16] I. Markovsky and P. Rapisarda, "Data-driven simulation and control," International Journal of Control, vol. 81, no. 12, pp. 1946-1959, 2008.

[17] I. Markovsky, J. C. Willems, P. Rapisarda, and B. L. De Moor, "Algorithms for deterministic balanced subspace identification," Automatica, vol. 41, no. 5, pp. 755-766, 2005.

[18] J. Björk, "Performance quantification of interarea oscillation damping using hvdc," Ph.D. dissertation, KTH Royal Institute of Technology, 2019.

[19] L. Huang, H. Xin, and Z. Wang, "Damping low-frequency oscillations through vsc-hvdc stations operated as virtual synchronous machines," IEEE Trans. Power Electron., 2018, early access.

[20] J. Coulson, J. Lygeros, and F. Dörfler, "Regularized and distributionally robust data-enabled predictive control," in 2019 IEEE Conference on Decision and Control (CDC), 2019, submitted.

[21] J. B. Jørgensen, J. K. Huusom, and J. B. Rawlings, "Finite horizon $\mathrm{mpc}$ for systems in innovation form," in 2011 50th IEEE Conference on Decision and Control and European Control Conference. IEEE, 2011, pp. 1896-1903.

[22] E. F. Camacho, C. Bordons, and M. Johnson, "Model predictive control. advanced textbooks in control and signal processing," 1999.

[23] L. Ljung, "System identification," in Signal analysis and prediction. Springer, 1998, pp. 163-173.

[24] J. Rocabert, A. Luna, F. Blaabjerg, and P. Rodriguez, "Control of power converters in ac microgrids," IEEE Trans. Power Electron., vol. 27, no. 11, pp. 4734-4749, 2012.

[25] L. Huang, J. Coulson, J. Lygeros, and F. Dorfler, "Data-enabled predictive control for grid-connected power converters," arXiv preprint arXiv:1903.07339, 2019.

[26] P. Kundur, N. J. Balu, and M. G. Lauby, Power system stability and control. McGraw-hill New York, 1994, vol. 7. 